\title{
Urinary neopterin in idiopathic retinal vasculitis
}

\author{
H E Palmer, G Giovannoni, M R Stanford, G R Wallace, E M Graham
}

\begin{abstract}
Aims-To determine whether urinary neopterin:creatinine (UNC) ratios relate to disease activity in idiopathic retinal vasculitis (RV).

Methods-18 patients with RV were prospectively recruited into a year long longitudinal study. Patients collected first morning urine samples on a weekly basis and on the same day completed a diary which documented their subjective view of RV activity and any concurrent infection. They were examined in clinic on a 6-8 weekly basis and an objective assessment was made of RV disease activity. 14 healthy controls collected urine samples in the same way.

Results-UNC ratios were significantly higher in patients than in controls ( $p=0.004$, Mann-Whitney $U$ test). UNC ratios were significantly higher when, according to their diaries, the patients had a subjective flare up of $\mathrm{RV} \quad(p=0.001$, Mann-Whitney $U$ test). Subjective increased RV activity occurred more often when the patients had a concurrent infection $\left(\mathbf{p}<0.0001, \chi^{2}\right.$ test $)$. There was no significant difference in the UNC ratio between objective clinical relapse and non-relapse of RV. There was moderate agreement between the clinical assessment and patients' subjective impression of $\mathrm{RV}$ activity $(\kappa=0.48)$.

Conclusions-Higher neopterin levels reflect cell mediated disease that occurs in RV, but UNC ratios are not recommended as a means of monitoring clinical disease activity in RV.

(Br F Ophthalmol 2001;85:30-33)
\end{abstract}

Medical Eye Unit, St

Thomas's Hospital, London SE1 7EH, UK H E Palmer

M R Stanford

E M Graham

Department of Ophthalmology H E Palmer

M R Stanford

G R Wallace

Institute of Neurology, Queen Square,

London, UK

G Giovannoni

Correspondence to: Miss Helen Palmer, Medical Eye Unit, St Thomas's Hospital, London SE1 7EH, UK

g.wallace@kcl.ac.uk

Accepted 23 June 2000 serum levels of soluble intercellular adhesion molecule 1 (sICAM-1) are known to be associ- could predict an imminent flare up. While ated with RV relapse, ${ }^{4}{ }^{5}$ regular monitoring of its levels in the clinical setting is not practicable.

Neopterin is a pyrazimo-pyrimidine derivative $^{6}$ that is used as a marker of disease activity in many inflammatory conditions and, as such, it has excited much recent interest. It is produced by human monocytes and macrophages in response to stimulation with interferon gamma $^{7}$ and, as it is relatively stable, it provides a useful marker of cell mediated immune activation. It is not catabolised, so its biological half life relies solely on renal clearance. ${ }^{8}$ This, together with the relative ease and cost efficiency for its analysis, makes neopterin a useful biological marker. ${ }^{9}$ For more accurate analysis, urinary neopterin concentrations are related to creatinine content to take account of physiological variations of urinary dilution. Thus the urinary neopterin:creatinine (UNC) ratio is normally used. Neopterin has a diurnal variation, with higher values at night. To obtain maximum levels for detection, it is therefore usual to analyse first morning urine samples. ${ }^{10}$

In a pilot study of $10 \mathrm{RV}$ patients with active disease who were off treatment, we found that not only were UNC ratios higher in patients than controls, but that UNC ratios correlated with sICAM-1 levels, ${ }^{11}$ a parameter that reflects disease activity. ${ }^{5}$ We therefore wished to investigate the role of the UNC ratio as a quick, easy, cheap and non-invasive marker of disease activity in RV; in particular, we wished to ascertain whether it would be of use as a predictor of imminent disease relapse.

\section{Methods}

CLINICAL STUDY

Patients with idiopathic RV were prospectively recruited from the medical eye unit for a 1 year period, having given their informed consent to enter the study. Idiopathic RV is characterised by retinal vascular leakage, cells in the vitreous, and is a condition which is apparently isolated to the eye, with no systemic inflammatory disease. Patients who were pregnant, those younger than 16 years of age, and those who were unable to give informed consent were not included in the study. A systemic inflammatory condition was excluded on the basis of history, systemic examination, and the following routine investigations: full blood count, erythrocyte sedimentation rate (ESR), urea and electrolytes, glucose, liver function test, serum angiotensin converting enzyme, and chest radiography. At each outpatient attendance a full ophthalmological assessment was made including Snellen and near vision acuities, slit lamp biomicroscopy, and indirect binocular ophthalmoscopic examination. RV disease ac- 
Table 1 Summary of UNCR results in patients with retinal vasculitis (RV) and control subjects

\begin{tabular}{llll}
\hline & $\begin{array}{l}\text { No. UNC ratio } \\
\text { counts }\end{array}$ & $\begin{array}{l}\text { Median (range) UNC } \\
\text { ratio }\end{array}$ & p valuet \\
\hline Controls & 383 & $133(49-674)$ & 0.01 \\
RV patients & 461 & $164(22-68236)$ & \\
Clinic (objective) RV activity & 13 & $179(47-1199)$ & 0.78 \\
$\quad$ Relapse & 68 & $138(16-53501)$ & \\
$\quad$ Non-relapse & & $170(16-1199)$ & 0.0005 \\
Diary (subjective) RV activity & 54 & $138(35-53501)$ & \\
$\quad$ Relapse & 407 & & \\
$\quad$ Stable & & $149(22-18013)$ & $<0.0001$ \\
$\quad$ Systemic treatment & 376 & $410(24-68236)$ & \\
$\quad$ Yes & 85 & & \\
$\quad$ No & & & \\
\hline
\end{tabular}

*Values omitted when concurrent infection noted in the subjects' diaries.

†Mann-Whitney U test. cence (353 nm excitation, $438 \mathrm{~nm}$ emission wavelengths). Creatinine concentration was analysed by UV absorption at $235 \mathrm{~nm}$ in the same chromatographic run to allow for variations in urinary density. ${ }^{10}$

STATISTICAL ANALYSIS

For analysis of UNC ratios, values were omitted when subjects had a concurrent infection so that differences in the ratio between different subject groups would only reflect RV in terms of disease activity. Differences in UNC ratio between groups were measured by the Mann-Whitney $U$ test. Comparison of agreement between objective and subjective assessments of RV activity was made by kappa analysis $(\kappa)^{13}$ : objective clinic examinations were compared with the most recent preceding diary entry. From the patients' diaries, $\chi^{2}$ analysis was used to investigate whether RV activity (relapse or non-relapse) was associated with the presence or absence of a concurrent infection.

\section{Results}

SUBJECT CHARACTERISTICS

Eighteen patients and 14 control subjects were recruited; half of each group were women. The age ranges in the patient and control groups were 20-61 years (mean 42) and 28-54 years (mean 39), respectively. There was no significant difference in age between the two groups.

With regard to treatment, three patients were off systemic treatment throughout the study, five were on prednisolone plus second line immunosuppressants, and 10 were taking prednisolone in doses ranging from $80 \mathrm{mg}$ to $2.5 \mathrm{mg}$.

The patients collected their urine samples over a mean period of 9 months (range $3-15.5)$. In the control group the mean period of collection was 7 months (range 3.25-12).

\section{UNC RATIOS}

Median UNC ratios are summarised in Table 1.

patients then brought the collected samples to the next outpatient visit which they attended at 6-8 weekly intervals, together with the completed diary for that time period.

The control subjects were healthy members of staff with no ocular or systemic disease. They collected first morning urine samples in the same way as the patients and kept a note of any concurrent infections.

\section{LABORATORY STUDIES}

Urinary neopterin levels were measured by reverse phase, high pressure liquid chromatography (HPLC). Aliquots of urine $(100 \mu \mathrm{l})$ were diluted and mixed with $1 \mathrm{ml}$ Sorensen potassium phosphate buffer containing $5.4 \mathrm{mmol} / 1$ disodium diaminoethylene tetraacetate to dissolve urinary sediments. Separation of analytes was performed with a column packed with $5 \mu \mathrm{m}$ reversed phase C-18 material. Chromatographic elution was performed with degassed Sorensen potassium phosphate buffer at a concentration of $15 \mathrm{mmol} / 1, \mathrm{pH} 6.4$. Neopterin was detected by its native fluores-

\section{Patients versus controls}

Median UNC ratios were 164 for patients with RV and 133 for controls, the ratio being significantly higher in the patient group than in controls ( $p<0.0001$, Mann-Whitney U test).

\section{Subjective RV activity (patients' diaries)}

From the patients' diaries corrected UNC levels were significantly higher when the patients felt subjectively that there was a flare up of RV (median UNC ratio 170 versus 138 for "stable" RV; p=0.0005, Mann-Whitney U test).

\section{Objective RV activity (examination at clinic} visits)

Of a total of 81 patient clinic visits, a relapse of RV was diagnosed on 13 occasions. There was no significant difference in the most recent preceding UNC ratio between relapse and non-relapse of RV (median levels 179 and 138, respectively) as judged objectively in the clinic ( $\mathrm{p}=0.78$, Mann-Whitney U test). 
Table 2 From the patients' diaries: comparison of presence or absence of infection with subjective RV activity

\begin{tabular}{llll}
\hline & \multicolumn{3}{l}{ Concurrent infection } \\
\cline { 2 - 4 } & No & Yes & Total \\
\hline Subjective RV activity & & & \\
$\quad$ Stable & 436 & 86 & 522 \\
Relapse & 58 & 50 & 108 \\
Total & 494 & 136 & 630 \\
\hline
\end{tabular}

$\mathrm{p}<0.0001$ ( $\chi^{2}$ test $)$.

Table 3 Frequencies of objective RV activity documented in clinic compared with patients'subjective assessment

\begin{tabular}{llll}
\hline & \multicolumn{3}{c}{ Subjective (patient diaries) } \\
\cline { 2 - 4 } & Stable & Relapse & Total \\
\hline Objective (clinical assessment) & & \\
Non-relapse & 65 & 7 & 72 \\
Relapse & 5 & 8 & 13 \\
Total & 70 & 15 & 85 \\
\hline$\kappa=0.48$. & & & \\
\hline
\end{tabular}

Treatment

The median UNC ratio was significantly lower in patients on systemic treatment (149) than in those who were off treatment $(410 ; \mathrm{p}<0.0001$, Mann-Whitney U test). There was no significant difference in levels between patients receiving prednisolone in a dose of $<20 \mathrm{mg} /$ day, prednisolone in a dose of $\geqslant 20 \mathrm{mg} /$ day, or prednisolone plus second line immunosuppressants.

\section{SUBJECTIVE ASSESSMENT OF RV ACTIVITY AND} CONCURRENT INFECTION

Of a total of 630 diary entries, patients felt that their disease was stable on 522 occasions $(82.9 \%$, Table 2$)$. When they recorded stable RV, only 86 of 522 entries (16.5\%) documented a concurrent infection. This was in contrast to 108 entries of subjective RV relapse $(17.1 \%)$ with a concurrent infection recorded in 50 of the 108 entries $(46.3 \%)$. Thus, there was significant association between stable RV and no concurrent infection, and between relapse of disease with an infection $(\mathrm{p}<0.0001$, $\chi^{2}$ test).

COMPARISON OF OBJECTIVE AND SUBJECTIVE ASSESSMENT OF DISEASE ACTIVITY

The observed frequencies of RV activity documented in the clinic (relapse or non-relapse) and those documented in the patients' most recent preceding diary entries (stable or relapse) are shown in Table 3. Of a total of 85 clinic visits, both patient and clinician agreed that the RV was stable at $65(76.5 \%)$ of the visits. When the clinician diagnosed a disease relapse (this occurred 13 times), the patient agreed in eight cases (61.5\%). Overall agreement between objective and subjective views of $\mathrm{RV}$ activity was moderately good $(\kappa=0.48)$.

\section{Discussion}

This is the first longitudinal study of urinary neopterin in patients with idiopathic RV; corrected urinary neopterin levels were found to be significantly higher in patients with RV than in controls. UNC ratios were higher when patients had a subjective flare up of disease documented in their diaries. There was a significant association between a subjective increase in RV activity and a concurrent infection, and moderate agreement between subjective and objective assessment of RV activity. There were only 13 episodes of objective clinical relapse throughout the study, and neopterin levels were not significantly different between clinical relapse and non-relapse.

Neopterin was first discovered in 1963 in bee larvae and 4 years later it was found in human urine. ${ }^{14}$ Since then raised levels have been found to be associated with a number of inflammatory and infectious human conditions. Urinary neopterin levels are potentially very useful for monitoring disease in the clinical setting as they provide a quick, cheap, and non-invasive test. Increased neopterin measurements occur in body fluids of recipients of solid grafts such as kidney ${ }^{15}$ and heart, ${ }^{16}$ and in bone marrow recipients levels are predictive of graft rejection. ${ }^{17}$ Urine and serum neopterin concentrations are increased in patients with rheumatoid arthritis, and levels correlate with both radiological stage and with disease activity. ${ }^{18}$ In thyroiditis neopterin concentrations differentiate autoimmune from nonautoimmune disease, being higher in the former. ${ }^{19}$ In systemic lupus erythematosus (SLE) levels are high in active disease..$^{20}$ Monitoring of neopterin in the clinical setting shows most potential in relation to HIV. In HIV-1 infections raised levels are usually seen several days before seroconversion, allowing a potentially useful screening method for donor blood that has negative HIV-1 antibody titres. ${ }^{21}$ The addition of neopterin values to the CD4+ count improves the predictive value for progression from HIV-1 infection to AIDS. ${ }^{22}$ Neopterin levels can also provide a useful prognostic index for survival time in HIV-1 infection. $^{23}$

Neopterin levels have previously been investigated in acute anterior uveitis. In that study, in contrast to our findings, levels did not differ between patients and controls, but were higher in patients with active disease. ${ }^{24}$ The researchers analysed serum levels rather than urinary levels of neopterin using a commercial immunoassay against which HPLC (used in our study) is considered the gold standard. In addition, direct determination of neopterin in serum is more difficult as its concentration is 500 times lower than in urine, which could also account for the discrepancy in findings between the two studies. ${ }^{25}$

The significantly raised levels of urinary neopterin found in patients with RV compared with controls further raises the question as to whether RV is an ocular manifestation of a systemic disease. This finding parallels that of serum sICAM-1, higher levels of which are also found in patients with RV. ${ }^{5}$ It is possible that the increased neopterin levels reflect cell mediated inflammatory activity in the eye alone, but extraocular neopterin production would also be possible. Since raised UNC ratios occur in patients with multiple sclero$\operatorname{sis}^{26}$ and as there is a known association between RV and multiple sclerosis, ${ }^{27}$ it is feasi- 
ble that the increased urinary neopterin levels recorded in some of these patients with apparently idiopathic disease was due to presymptomatic or subclinical multiple sclerosis. Likewise, some of these patients could have a systemic inflammatory disease such as sarcoidosis. $^{28}$

We have found that UNC ratios in RV were not significantly raised in clinical relapse, although there were only 13 episodes of clinical relapse for all of the patients which limits the power of the analysis. At present, therefore, the case for using urinary neopterin levels as a means of monitoring this disease is not proved but merits further investigation. Neopterin levels were, however, significantly higher when the patients subjectively felt they had a flare up of RV. This, together with the moderate agreement between objective and subjective disease activity, suggests that patients are good monitors of their disease.

Urinary neopterin levels were lower when patients were on treatment. Neopterin is produced by macrophages in response to stimulation primarily by the cytokine interferon gamma (IFN $\gamma$ ), whilst tumour necrosis factor alpha $(\mathrm{TNF} \alpha)$ synergistically amplifies its production. ${ }^{7}$ Since glucocorticoids reduce production of these two cytokines, ${ }^{29}$ it is not surprising that oral prednisolone reduces neopterin levels. This finding is consistent with other studies in which prednisolone has been shown to reduce neopterin. In HIV positive patients with wasting disease, a reduction in neopterin is seen 7 days after starting oral prednisolone. ${ }^{30}$ In both rheumatoid arthritis and SLE a fall in neopterin occurs when patients show a reduction in disease activity in response to immunosuppressive treatment. ${ }^{18} 20$ It is interesting that there was no difference in UNC ratios between patients on different doses of prednisolone, nor between those on additional second line immunosuppressants. This may be because some patients required more treatment than others to bring their disease activity under similar control.

In conclusion, while UNC ratios are not recommended as a means of monitoring disease activity in RV, this study has shown for the first time that higher levels of urinary neopterin occur in patients with RV than in controls. This reflects immune activation which occurs with this disease and gives further evidence that idiopathic RV could be an ocular manifestation of a systemic disease.

This research was funded by the St Thomas' Hospital Endowments Committee, the Iris Fund for the Prevention of Blindness, and the Multiple Sclerosis Society of Great Britain \& Northern Ireland.

1 Rothova A, Suttorp-Schulten MSA, Treffers WF, et al. Causes and frequency of blindness in patients with intraocular inflammatory disease. $\mathrm{Br} \quad \mathcal{f}$ Ophthalmol 1996;80:332-6.
2 Forrester JV. Uveitis: pathogenesis Lancet 1991:338:1498 501.

3 Lightman S. Uveitis: management. Lancet 1991;338:15014.

4 Zaman AG, Edelsten C, Stanford MR, et al. Soluble intercellular adhesion molecule-1 (sICAM-1) as a marker of disease relapse in idiopathic uveoretinitis. Clin Exp Immunol 1994;95:60-5.

5 Palmer HE, Zaman AG, Ellis BA, et al. Longitudinal analysis of soluble intercellular adhesion molecule-1 in retinal vasculitis patients. Eur 7 Clin Invest 1996;26:686-91.

6 Brown GM. The biosynthesis of pteridines. Adv Enzymol 1971;35:5-77.

7 Huber C, Batchelor JR, Fuchs D, et al. Immune responseassociated production of neopterin. Release from macrophages primarily under control of interferon gamma. $\mathcal{F}$ Exp Med 1984;160:310-6.

8 Fuchs D, Weiss G, Wachter H. Neopterin, biochemistry and clinical use as a marker for cellular immune reactions. Int Arch Allergy Immunol 1993;101:1-6.

9 Wachter H, Fuchs D, Hausen A. Neopterin as a marker for activation of cellular immunity: immunologic basis and application. Adv Clin Chem 1989;27:81-141.

10 Fuchs D, Hausen A, Reibnegger G, et al. Automatized routine estimation of neopterin in human urine by HPLC on reversed phase. In: Wachter H, Curtius H-C, Pfleiderer W, eds. Biochemical and clinical aspects of pteridines. Berlin, New York: de Gruyter, 1982:67-79.

11 Palmer HE, Giovannoni G, Stanford MR, et al. Urinary neopterin:creatinine ratios correlate with serum sICAM-1 levels in idiopathic retinal vasculitis. Vision Res 1995:35: S106.

12 Stanford MR, Graham EM, Kasp E, et al. A longitudinal study of clinical and immunological findings in 52 patients with relapsing retinal vasculitis. Br $\mathcal{F}$ Ophthalmol 1988;72: $442-7$.

13 Altman DG. Practical statistics for medical research. 1st ed. London: Chapman \& Hall, 1991:403-5.

14 Sakurai A, Goto M. Neopterin: isolation from human urine. f Biochem (Tokyo) 1967;61:142-5.

15 Margreiter R, Fuchs D, Hausen A, et al. Neopterin as a new biochemical marker for diagnosis of allograft rejection. Experience based upon evaluation of 100 consecutive cases. Transplantation 1983;36:650-3.

16 Muller TF, Vogl M, Neumann MC, et al. Noninvasive monitoring using serum amyloid A and serum neopterin in cardiac transplantation. Clin Chim Acta 1998;276:63-74.

17 Niederwieser D, Huber C, Gratwohl A, et al. Neopterin as a new biochemical marker in the clinical monitoring of bone marrow transplant recipients. Transplantation 1984;38:497500.

18 Reibnegger G, Egg D, Fuchs D, et al. Urinary neopterin reflects clinical activity in patients with rheumatoid arthritis. Arthritis Rheum 1986;29:1063-70.

19 Schwedes U, Teuber J, Schmidt R, et al. Neopterin as a marker for the activity of autoimmune thyroid diseases. marker for the activity of auto
Acta Endocrinol 1986;111:51-2.

20 Leohirun L, Thuvasethakul P, Sumethkul V, et al. Urinary neopterin in patients with systemic lupus erythematosus. Clin Chem 1991;37:47-50.

21 Zangerle R, Schonitzer D, Fuchs D, et al. Reducing HIV transmission by seronegative blood. Lancet 1992;339:130-

22 Planella T, Cortes M, Martinez-Bru C, et al. The predictive value of several markers in the progression to acquired immunodeficiency syndrome. Clin Chem Lab Med 1998;36: 169-73.

23 Rogstad KE, Morgan L, Hughes J. Urinary neopterin as a prognostic index of survival time in HIV-1 infection. Int $\mathcal{F}$ STD AIDS 1998:9:326-9.

24 Abi-Hanna D, Wakefield D. Increased serum neopterin levels in patients with acute anterior uveitis. Curr Eye Res 1988;7:497-502.

25 Werner ER, Bichler A, Daxenbichler G, et al. Simultaneous determination of neopterin and creatinine in serum with solid phase extraction and on-line elution liquid chromatography. Clin Chem 1987;33:2028-33.

26 Giovannoni G, Lai M, Kidd D, et al. Daily urinary neopterin excretion as an immunological marker of disease activity in multiple sclerosis. Brain 1997;120:1-13.

27 Stanford MR, Graham EM. Systemic associations of retinal Stanford MR, Graham EM. Systemic associations

28 Eklund A, Blaschke E. Elevated serum neopterin levels in sarcoidosis. Lung 1986;164:325-32.

29 Ramirez F, Fowell DJ, Puklavec M, et al. Glucocorticoids promote a Th2 cytokine response by CD $4+\mathrm{T}$ cells in vitro. f Immunol 1996;156:2406-12.

30 Kilby JM, Tabereaux PB, Mulanovich V, et al. Effects of tapering doses of oral prednisolone on viral load among HIV-infected patients with unexplained weight loss. AIDS Res Hum Retroviruses 1997;13:1533-7. 